\title{
ВMJ Global Health Costs of hand hygiene for all in household settings: estimating the price tag for the 46 least developed countries
}

\author{
Ian Ross (D) , J Joanna Esteves Mills, ${ }^{2}$ Tom Slaymaker, ${ }^{3}$ Richard Johnston, ${ }^{2}$ \\ Guy Hutton, ${ }^{3}$ Robert Dreibelbis, ${ }^{1}$ Maggie Montgomery ${ }^{2}$
}

To cite: Ross I, Esteves Mills J, Slaymaker T, et al. Costs of hand hygiene for all in household settings: estimating the price tag for the 46 least developed countries. BMJ Global Health 2021;6:e007361. doi:10.1136/ bmjgh-2021-007361

Handling editor Edwine Barasa

- Additional supplemental material is published online only. To view, please visit the journal online (http://dx.doi.org/10. 1136/bmjgh-2021-007361)

Received 6 September 2021 Accepted 19 November 2021

Check for updates

(c) Author(s) (or their employer(s)) 2021. Re-use permitted under CC BY. Published by BMJ.

${ }^{1}$ Department of Disease Control London School of Hygiene \& Tropical Medicine, London, UK ${ }^{2}$ World Health Organization, Geneva, Switzerland ${ }^{3}$ UNICEF, New York, New York, USA

Correspondence to

Dr lan Ross;

ian.ross@Ishtm.ac.uk

\section{ABSTRACT}

Introduction Domestic hand hygiene could prevent over 500000 attributable deaths per year, but 6 in 10 people in least developed countries (LDCs) do not have a handwashing facility (HWF) with soap and water available at home. We estimated the economic costs of universal access to basic hand hygiene services in household settings in 46 LDCs.

Methods Our model combines quantities of households with no HWF and prices of promotion campaigns, HWFs, soap and water. For quantities, we used estimates from the WHO/UNICEF Joint Monitoring Programme. For prices, we collated data from recent impact evaluations and electronic searches. Accounting for inflation and purchasing power, we calculated costs over 2021-2030, and estimated total cost probabilistically using Monte Carlo simulation.

Results An estimated US\$12.2-US\$15.3 billion over 10 years is needed for universal hand hygiene in household settings in 46 LDCs. The average annual cost of hand hygiene promotion is US\$334 million (24\% of annual total), with a further US\$233 million for 'top-up' promotion (17\%). Together, these promotion costs represent US\$0.47 annually per head of LDC population. The annual cost of HWFs, a purpose-built drum with tap and stand, is US\$174 million (13\%). The annual cost of soap is US\$497 million (36\%) and water US\$127 million (9\%). Conclusion The annual cost of behavioural change promotion to those with no HWF represents $4.7 \%$ of median government health expenditure in LDCs, and $1 \%$ of their annual aid receipts. These costs could be covered by mobilising resources from across government and partners, and could be reduced by harnessing economies of scale and integrating hand hygiene with other behavioural change campaigns where appropriate. Innovation is required to make soap more affordable and available for the poorest households.

\section{INTRODUCTION}

Hand hygiene reduces transmission of a variety of enteric and respiratory infections. ${ }^{12}$ Every year, 165000 deaths from diarrhoeal disease and 370000 deaths from acute respiratory infections are attributable to inadequate hand hygiene. ${ }^{3}$ However, nearly a third of the global population do not have

\section{Key questions}

What is already known?

- Understanding resource requirements is important for planning, but data on the costs of improving domestic hand hygiene are scarce.

- While a 2016 study estimated the global cost of drinking water, sanitation and hygiene, it did not report hygiene-specific estimates of recurrent or total cost, nor did it describe the assumed promotion intervention and handwashing facility or consider alternatives to them.

What are the new findings?

- The total economic cost over 10 years is US\$12.2US $\$ 15.3$ billion, of which US\$4.9-US\$6.6 billion $(42 \%)$ is for behavioural change promotion interventions.

- The remainder is for facilities and supplies, with soap the biggest cost category $(36 \%)$, followed by handwashing facilities (13\%) and water (9\%).

- The facility and supply costs per household comprise an initial investment in a handwashing facility (lasting 5 years) at a median of US\$17, accompanied by an annual cost of US\$17 for soap and US\$5 for water.

What do the new findings imply?

- The annual cost of behavioural change promotion to those with no handwashing facility represents $4.7 \%$ of median government health expenditure in least developed countries

- On top of this, investments in infrastructure and supplies are required. Soap in particular is a substantial and recurrent cost, which may be unaffordable for the poorest households.

- Promotion costs could be covered by mobilising resources from across government and partners, and could be reduced by harnessing economies of scale and integrating hand hygiene with other behavioural change campaigns where appropriate.

handwashing facilities (HWFs) with soap and water available at home, denoted a 'basic' hygiene service. ${ }^{4}$ Many more do not practice handwashing with soap at critical times-for example, only $26 \%$ of potential faecal contact 
events globally are followed by handwashing with soap. ${ }^{5}$ The COVID-19 pandemic highlighted the need for hand hygiene to reduce transmission across settings, including households, schools, healthcare facilities and public places. ${ }^{6}$

The greatest deficit is in least developed countries (LDCs), where 6 in 10 people are without a basic hygiene service, of which about half have a HWF but no soap and/or water. ${ }^{4}$ However, promotion of handwashing behaviours is required in all countries and settings. For example, it is estimated that in high-income countries, where over $99 \%$ of the population have water accessible on premises, ${ }^{4}$ only $51 \%$ of faecal contacts are followed by handwashing with soap. ${ }^{5}$ Hand hygiene is best facilitated by an on-premises water supply, ${ }^{7}$ but $60 \%$ of the LDC population do not have such a service, ${ }^{4}$ instead hauling water from off-premises sources.

Reviews of factors for success in scaling up public health interventions in low-income and middle-income countries (LMICs) frequently identify costing and economic analysis of interventions in the top three success factors. ${ }^{89}$ For hand hygiene, such cost figures are scarce. A 2016 study costing the Sustainable Development Goal (SDG) targets for drinking water, sanitation and hygiene (WASH) in 140 countries by Hutton and Varughese identified only five peer-reviewed studies providing data on the costs of handwashing promotion, alongside seven from grey literature. ${ }^{1011}$ The absence of robust cost data for handwashing programmes makes financial planning and resource allocation difficult. ${ }^{12}$

Hutton and Varughese reported an annual capital cost of US $\$ 2.0$ billion over 15 years for 140 LMICs to achieve basic hand hygiene in domestic settings. ${ }^{10} 11$ This figure includes HWFs and promotion only. Limitations of these cost estimates include: (1) not reporting hygiene-specific estimates of recurrent or total costs, only incorporating them into totals for WASH; (2) no description of the assumed hygiene promotion 'software' or the assumed HWF type(s); (3) inconsistent approach to HWF useful life, with 10 years applied for rural areas in all countries, but 2.5 or 5 years for urban areas in $86 \%$ of countries; (4) no sensitivity analysis specific to hygiene assumptions.

The costs of activities promoting hand hygiene in LDCs are typically borne by governments and donors as a public health investment. However, only $16 \%$ of the 115 countries responding to a WHO-led survey could report the size of hygiene budgets or expenditures, compared with $53 \%$ for sanitation and/or water supply. ${ }^{13}$ Of the 16 countries providing expenditure data, 8 indicated only one source of funding (government, households or donors), indicating that the data are not comprehensive. ${ }^{13}$ While the costs of HWFs, soap and water are borne by households in the majority of cases, they can also be subsidised directly or indirectly (eg, specific subsidies, cash transfers or humanitarian response). Affordability is a concern, and survey data suggest a strong socio-economic gradient in soap availability within households. ${ }^{14}$
In this study, we aim to estimate the economic costs of universal access to basic hand hygiene services in household settings in 46 LDCs. Underlying objectives were to facilitate discussions and plans at the national and global levels, especially in light of the ongoing COVID-19 pandemic, and evaluate the size of the cost in relation to other investment priorities.

\section{METHODS}

We built a model combining quantities of targeted households with prices of capital and recurrent items, to estimate the economic costs of basic hand hygiene in domestic settings in 46 LDCs (2021 list - online supplemental material A) ${ }^{15}$ We followed the reference case of the Global Health Cost Consortium. ${ }^{12}$ The model structure is visualised in online supplemental material B.

\section{Costing perspective and approach}

We estimated economic costs from a societal perspective, and address who might bear those costs in the discussion section. We model straight-line scale-up of a hand hygiene promotion intervention (described below) over a 10-year horizon (2021-2030), whereby 10 equal cohorts of unserved households per country receive the intervention per year. Each cohort starts incurring recurrent costs in the year they receive the intervention. The scope of costed inputs comprised all activities contributing to behavioural change and purchase/use of a HWF over its useful life. We analysed quantities and prices per country for the 46 LDCs, separately for urban/rural areas. We then aggregated to an LDC total. Following norms in resource requirement estimation, we estimate the cost of reaching all target households, and do not incorporate the effectiveness of interventions. ${ }^{12}$

\section{Data on quantities}

We retrieved hygiene service level estimates for the 46 LDCs from the WHO/UNICEF Joint Monitoring Programme (JMP) ${ }^{16}$ For countries missing JMP estimates for 2020 (online supplemental material B), we applied an earlier year wherever possible (six countries). In the absence of any data we applied the LDC average (five countries). We calculated the number of households to be targeted per country by urban and rural setting, based on: (1) JMP coverage estimates; (2) average household sizes for urban/rural from the latest Demographic and Health Survey ${ }^{17}$; (3) UN medium-variant population projections $^{18}$; (4) assuming one HWF per household (equation in online supplemental material B). For global monitoring the JMP only counts HWFs that are located within the dwelling, yard or plot. HWFs may be fixed or mobile, and include a sink with tap, bucket with taps, or jug designated for handwashing. ${ }^{4}$ On-premises water supplies, for example, a private tubewell, could also be considered a HWF if survey respondents indicate that that is where they most often wash their hands.

For our headline result (A), the population for which interventions are costed comprises households with 
'no HWF'. This is because the relevant SDG indicator focuses on universal access to a HWF with soap and water on premises, rather than hygiene behaviour. However, we separately estimate two other results: (B) soap and water costs for households with a 'limited' service (HWF observed but soap and/or water missing); (C) promotion costs if the whole LDC population is targeted regardless of JMP status. The latter may be required because, even though handwashing with soap is twice as likely to be practised when a designated HWF is present, ${ }^{5}$ overall prevalence remains low. Our headline result (A) may underestimate promotion costs if populations beyond those without HWFs are to be targeted, and it is likely to be more practical to deliver behaviour change promotion to whole communities.

There were 61 million households in LDCs with 'no hygiene service' in 2020 ( $71 \%$ of which are in rural areas), and a further 81 million with a 'limited' service (69\% in rural areas). We used JMP analysis of the latest household survey containing HWF observation data for 42 of the 46 LDCs. ${ }^{4}$ We divided a country's population with a 'limited' service into three categories based on the limiting factor(s): missing soap only (47\% on average), missing water only (10\%) and missing both $(43 \%)$. We attribute the appropriate recurrent costs for those in the 'limited' group based on the split per country.

\section{Data on prices}

We collated price data from studies included in an earlier review, ${ }^{10}$ electronic searches (online supplemental material B), and by contacting impact evaluation investigators. For the latter, we contacted corresponding authors of 35 handwashing impact evaluations published since 2012, targeting those included in recent systematic reviews, ${ }^{11920}$ asking for cost data regarding the interventions they evaluated. For the cost of promotion, we identified 14 interventions (online supplemental material B) ${ }^{21-32}$ For the cost of HWFs and annual expenditure on soap for handwashing, we identified 16 and 10 data points respectively. In addition, we were able to estimate the cost of formative research for five of the 14 promotion interventions, for which the 2019 US $\$$ mean was about US $\$ 100000 .^{212225-27}$

For the cost of water for handwashing, we estimated an average annual cost separately for urban/ rural areas. This was based on the proportion of the population using piped improved water supplies per country, ${ }^{4}$ and assumptions about the economic cost of water for piped and non-piped users based on average national tariffs, and water volumes used for handwashing (online supplemental material B).$^{33}$ This aims to represent the recurrent cost of water from existing supplies. The additional capital costs of providing new supplies to those who still lack improved drinking water sources were not included.

\section{Intervention definition}

There are many possible approaches to promoting uptake of hand hygiene behaviours and facilities-no pre-existing intervention typology is universally used and no single programmatic approach is dominant. ${ }^{20} \mathrm{We}$ aimed to estimate costs of an intervention representing 'normative best practice ${ }^{12}$ to the extent possible in this context. This was defined in consultation with an expert steering group and based on the types of interventions prevalent in the available price data. We characterised the intervention in the base case as a hygiene promotion campaign with modes of delivery including one-to-one promotion (eg, house-to-house visits), group activities (eg, community meetings, roadshows, street theatre) and mass media (eg, radio, television, social). We assume that it is preceded by formative research to identify target drivers of behaviours and the development of a comprehensive behaviour change strategy. ${ }^{34}$

\section{Relation of intervention characteristics to effectiveness and price}

We know of only one review exploring whether more intensive interventions have greater effects on health or behaviour. Pickering et al undertook a systematic review of the impact of handwashing interventions on diarrhoea, focusing on the characteristics of studies which did and did not report a statistically significant reduction in diarrhoea. ${ }^{35}$ Of the 10 identified handwashing studies which reported frequency of contact between promoter and participant, none of the 3 which contacted participants once per month reported a significant effect on diarrhoea. Among the remaining seven which contacted participants twice a month or more, five reported a significant effect on diarrhoea (of which three contacted twice a week or more). Similar findings were reported for point-of-use water treatment. ${ }^{35}$ It is not possible to say whether the additional cost of more intensive interventions justifies increased likelihood of health effects, since there is only one primary cost-effectiveness study of a hygiene intervention in an LMIC domestic setting. ${ }^{21} 36$

In support of intervention definition for our study, IR and JEM extracted data on characteristics of the 14 promotion interventions for which we had cost data, including: the nature of formative research; theoretical basis ${ }^{20}$; modes of delivery; scale; and frequency (eg, number of visits/meetings). Categorising interventions by these characteristics, we assessed the relationship between each characteristic and price per household (to the extent possible, given missing data). Other than scale, the design-relevant characteristic which appeared highly correlated with price per household was modes of delivery employed, particularly whether one-to-one promotion activities (eg, household visits by a promoter) were included. Given the apparent higher effectiveness of more intensive interventions, ${ }^{35}$ we include one-to-one promotion in the intervention modelled in our 'base case'. However, we also model a lower-cost 'alternative case' which excludes one-to-one promotion. We present strip plots of input price variables (figure 1), and tabulate the underlying data in online supplemental material C. 


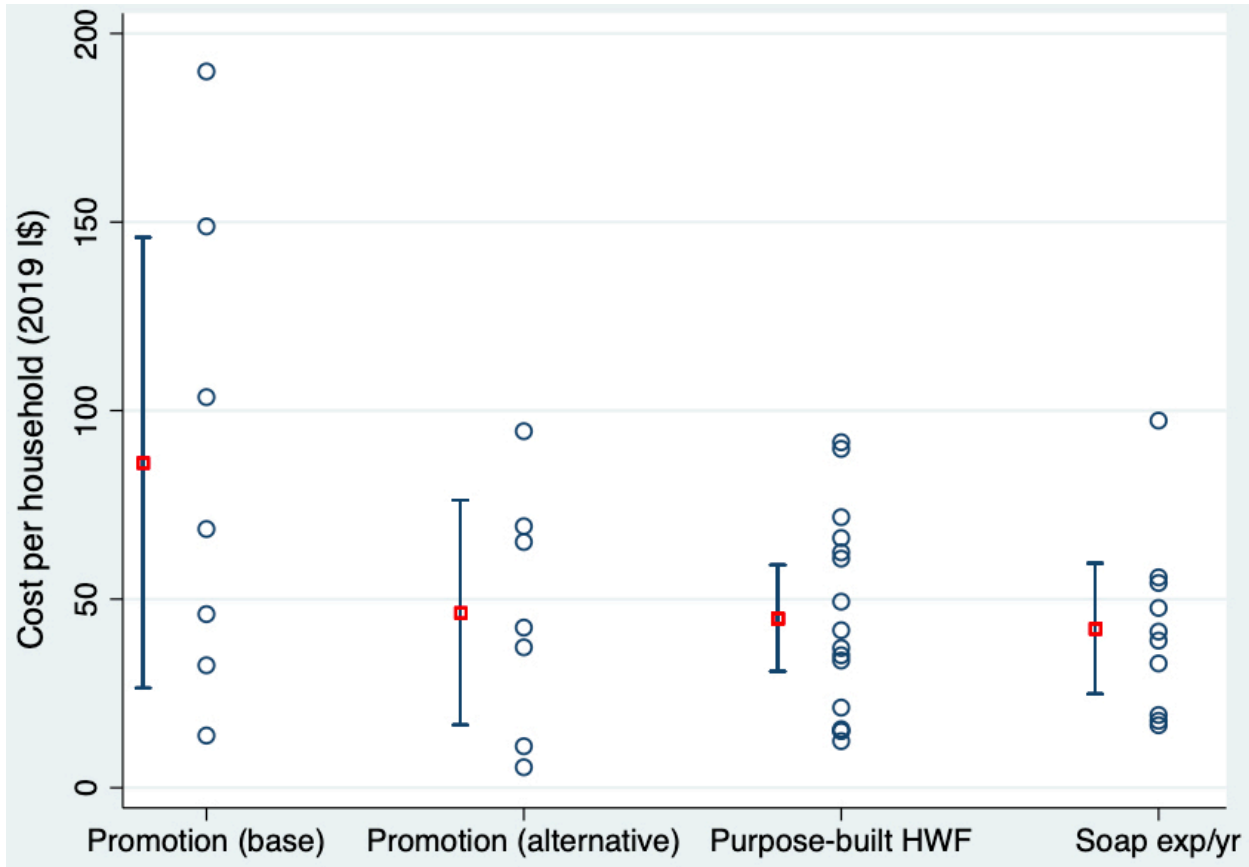

Figure 1 Distribution of key input price variables in 2019 international dollars (I\$). Red square with bars denotes $\mid \$$ mean and $95 \% \mathrm{Cl}$. Blue dots denote datapoints. 'Promotion (base)' is the base case intervention including one-to-one activities, group activities and mass media. 'Promotion (alternative)' is the case excluding one-to-one activities. This figure presents results in $I \$$, to illustrate the shape of data from which means were calculated, and which are directly comparable. Median US\$ prices per household after converting to local purchasing power are US\$33.9 for base case promotion, US\$14.8 for HWF, US\$14.8 for soap expenditure and US\$19.4 for alternative case promotion. Median annual expenditure per household on water for handwashing was US\$5.7 in rural areas and US\$4.0 in urban areas. The US\$ median for the home-made HWF considered under sensitivity analysis was \$1.2. HWF, handwashing facility.

It was not possible to account for scale empirically, with too few datapoints for larger-scale interventions and the larger-scale interventions also tending to include fewer modes of delivery. In the base case, we applied the mean of prices from studies of interventions including one-to-one promotion, which represents interventions delivered at various scales. We model assumptions for economies of scale in sensitivity analysis.

\section{Cost categories}

There are six cost categories (table 1). Conceptualising promotion as a software capital investment, its useful life is assumed to be 5 years, after which it is repeated in full. 'Top-up' promotion occurs annually, based on the theory that messages need to be repeatedly reinforced for behavioural change to be sustained. Following the intervention, all households are assumed to acquire a HWF. Thereafter, recurrent costs of supplying soap and water are incurred.

\section{Statistical analysis and sensitivity analysis}

We annuitised capital costs and discounted all costs at 3\% in the base case following reference case guidance. ${ }^{12} 37$ We report results in 2019 US dollars (US\$). We analysed

\begin{tabular}{|c|c|}
\hline Cost category & Description and key assumptions \\
\hline $\begin{array}{l}\text { 1. Formative research } \\
\text { (software capital) }\end{array}$ & $\begin{array}{l}\text { Design-focused research and piloting to identify target drivers of behaviours and the development of a } \\
\text { comprehensive behaviour change strategy. It is conceptualised as a one-off investment with useful life of } 1 \text { year. }\end{array}$ \\
\hline $\begin{array}{l}\text { 2. Promotion (software } \\
\text { capital) }\end{array}$ & $\begin{array}{l}\text { Hygiene promotion campaign with a useful life of } 5 \text { years, and modes of delivery including: (1) house-to-house } \\
\text { visits by promoters; (2) community/group activities and (3) mass media (online supplemental material B). }\end{array}$ \\
\hline $\begin{array}{l}\text { 3. Handwashing facility } \\
\text { (hardware capital) }\end{array}$ & $\begin{array}{l}\text { Purpose-built } 20 \text { litre drum with tap, basin and stand, with a useful life of } 5 \text { years. No capital maintenance is } \\
\text { assumed, due to short useful life and very simple infrastructure. }\end{array}$ \\
\hline $\begin{array}{l}\text { 4. Top-up promotion } \\
\text { (software capital } \\
\text { maintenance) }\end{array}$ & $\begin{array}{l}\text { Additional promotion activities occurring annually at } 25 \% \text { of initial cost (an assumption based on expert } \\
\text { judgement), representing a lighter version of the intervention with lower frequency and dose }\end{array}$ \\
\hline 5. Soap (recurrent) & $\begin{array}{l}\text { Expenditure on soap for handwashing (ie, excluding other uses). The type of soap varied across studies but was } \\
\text { predominantly bar soap. }\end{array}$ \\
\hline 6. Water (recurrent) & $\begin{array}{l}\text { Expenditure on water used for handwashing, assumed to average } 1.5 \mathrm{~L} / \text { person/day (online supplemental material } \\
\text { B). }{ }^{47}\end{array}$ \\
\hline
\end{tabular}


prices by first converting to 2019 prices in local currency to adjust for inflation. ${ }^{38}$ We then converted to 2019 international dollars (I\$), using World Bank data on GDP deflators and purchasing power conversion. ${ }^{39}$ After estimating the I\$ mean and standard error (SE) per cost category for available data, we converted them to 2019 US\$ per country. The rationale for converting to I\$ to take the mean is that the US\$ mean would be biased by purchasing power. For example, the I $\$$ mean for annual soap expenditure in our data is $\mathbf{I} \$ 42$, while the equivalent US\$ value is US\$10 at Afghanistan's purchasing power, or US\$18 in Niger.

All parameters in models are uncertain, but some are based on empirical data while others are the analyst's assumptions. Interventions costed in the studies underlying our price estimates varied, for example, in their modes of delivery, programme design, and scale. We sought to characterise parameter uncertainty using probabilistic sensitivity analysis, by taking a Bayesian approach using repeated draws from prior distributions constructed from available price data. We developed a probabilistic estimate of total cost using 1000 draws in a Monte Carlo simulation, calculating a 95\% uncertainty interval based on iterations' percentiles. Price data for all cost categories were right-skewed so were modelled as gamma distributed, in line with usual practice. ${ }^{40}$ In the absence of published CIs for JMP data, quantities were not varied probabilistically. We also undertook deterministic sensitivity analysis to explore the impact of uncertainty surrounding individual parameters and assumptions (scenarios in online supplemental material D). We present tornado plots indicating the magnitude of changes in total cost when parameters are at high and low plausible values.

\section{Patient and public involvement}

Public involvement was not undertaken as the study analysed secondary data from 46 countries.

\section{RESULTS}

In the base case, the total economic cost of hand hygiene for all in household settings in 46 LDCs over 10 years was between US $\$ 12.2$ and US $\$ 15.3$ billion $(95 \% \mathrm{CI}$, point estimate 13.7 billion) (figure 2). The cost of initial hand hygiene promotion was US $\$ 334$ million per year on average (24\% of total costs), with a further $\$ 233$ million per year for 'top-up' promotion (17\%). The cost of HWFs, a purpose-built drum with tap and stand/basin per household, was US $\$ 174$ million per year $(13 \%)$. The cost of supplies was US $\$ 497$ million per year for soap $(36 \%)$, and US\$127 million for water $(9 \%)$.

Considering only promotion costs, the total cost was US $\$ 5.7$ billion (95\% CI 4.9 to 6.6 ), or US $\$ 0.47$ annually per head of LDC population ( $42 \%$ of the total). Considering only HWFs and supplies, the total was US $\$ 8.0$ billion (95\% CI: 7.3 to 8.7), or US $\$ 0.66$ annually per head of LDC population $(58 \%$ of the total). An alternative framing of the facility and supply costs-considering cost per household-is an initial investment in a HWF (lasting 5 years) at a median of US $\$ 17$, accompanied by an annual cost of US $\$ 17$ for soap and US $\$ 5$ for water (Figure).

Different cost categories change in relative importance over time, as the intervention is scaled up over 10 years (figure 3). Capital costs, specifically the initial promotion and HWF, are consistent over the years as each of the 10 cohorts receives the initial intervention-the slight annual decrease is due to discounting. Total recurrent costs increase every year, which is due to new cohorts receiving top-up promotion and spending on soap and water. Overall, capital costs comprise $37 \%$ of the 10 -year total and recurrent costs $63 \%$, recalling that top-up promotion is a recurrent cost (table 1). In the alternative scenario where one-to-one promotion was excluded from the intervention, total cost decreased to US\$ 11.0 billion (95\% CI 10.0 to 12.2 ) and promotion cost to US $\$ 3.0$ billion (95\% CI 2.7 to 3.5 ), or US $\$ 0.25$ annually

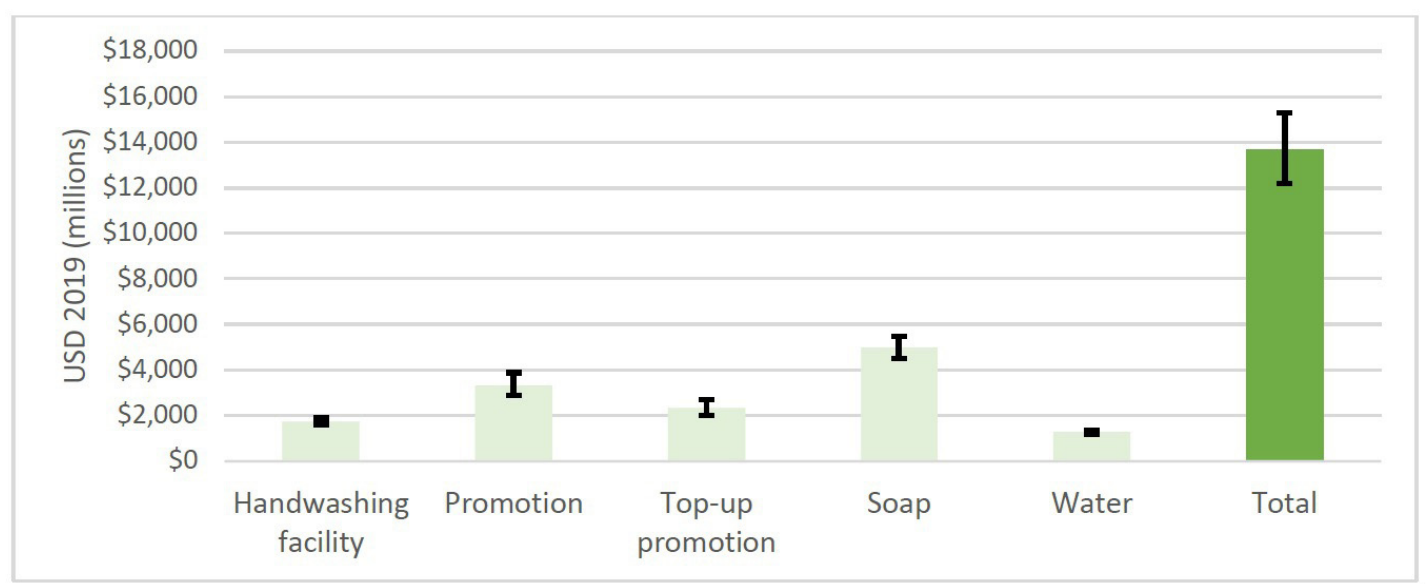

Figure 2 Total economic cost over 10 years of hand hygiene for all in domestic settings in 46 least developed countries (US\$). Error bars represent the $95 \% \mathrm{Cl}$ of 1000 draws in Monte Carlo simulation. The cost of initial formative research (US\$ 5 million) is not shown. 


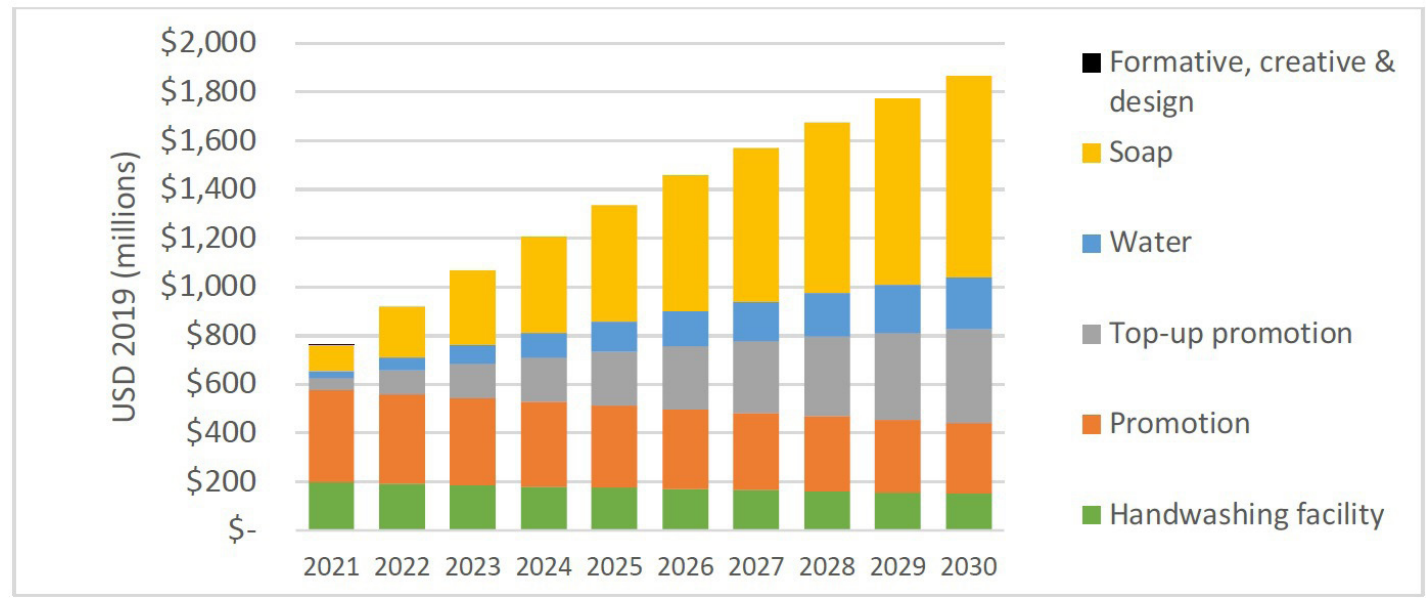

Figure 3 Distribution of costs over the 10-year time horizon, for the 46 least developed countries.

per head of LDC population- figures in online supplemental material D.

Considering the first of the additional results, the annual cost of soap and water enabling households with a 'limited' service to reach 'basic' would be US $\$ 1.4$ billion, of which $86 \%$ is for soap. Second, if all households in LDCs were targeted with hygiene promotion instead of only those with no HWF, the promotion cost almost quadruples to US $\$ 20.5$ billion (from US $\$ 5.7$ billion) including one-to-one activities, and to US $\$ 10.4$ billion excluding them (from US $\$ 3.0$ billion). These amounts are not included in the headline totals.

In deterministic sensitivity analysis, the scenario which saw the biggest difference in total cost was when the promotion price was varied according to its $95 \%$ CI. This saw total cost fall to US $\$ 10.5$ billion or rise to US $\$ 16.8$ billion (figure 4). Incorporating assumptions about economies of scale saw total cost falling to US $\$ 10.5$ billion (figure 4 ).
In this scenario, the prices of promotion, HWFs and soap experience annual decreases starting with $10 \%$ in year 2 then in $2 \%$ decrements ( $8 \%$ in year 3 , etc). All scenarios are described in online supplemental material $\mathrm{D}$, as are results for sensitivity analysis for promotion costs only.

\section{DISCUSSION}

Our study represents the most detailed to date of the costs of hand hygiene in domestic settings. We built on an earlier global study ${ }^{10}$ by differentiating between intervention modes of delivery and HWF technology through extracting study characteristics, by modelling alternative scenarios for these, and by characterising the uncertainty deriving from hygiene-related assumptions and price data. We also obtained far more price datapoints by contacting impact evaluation investigators and conducting new searches. Our application of

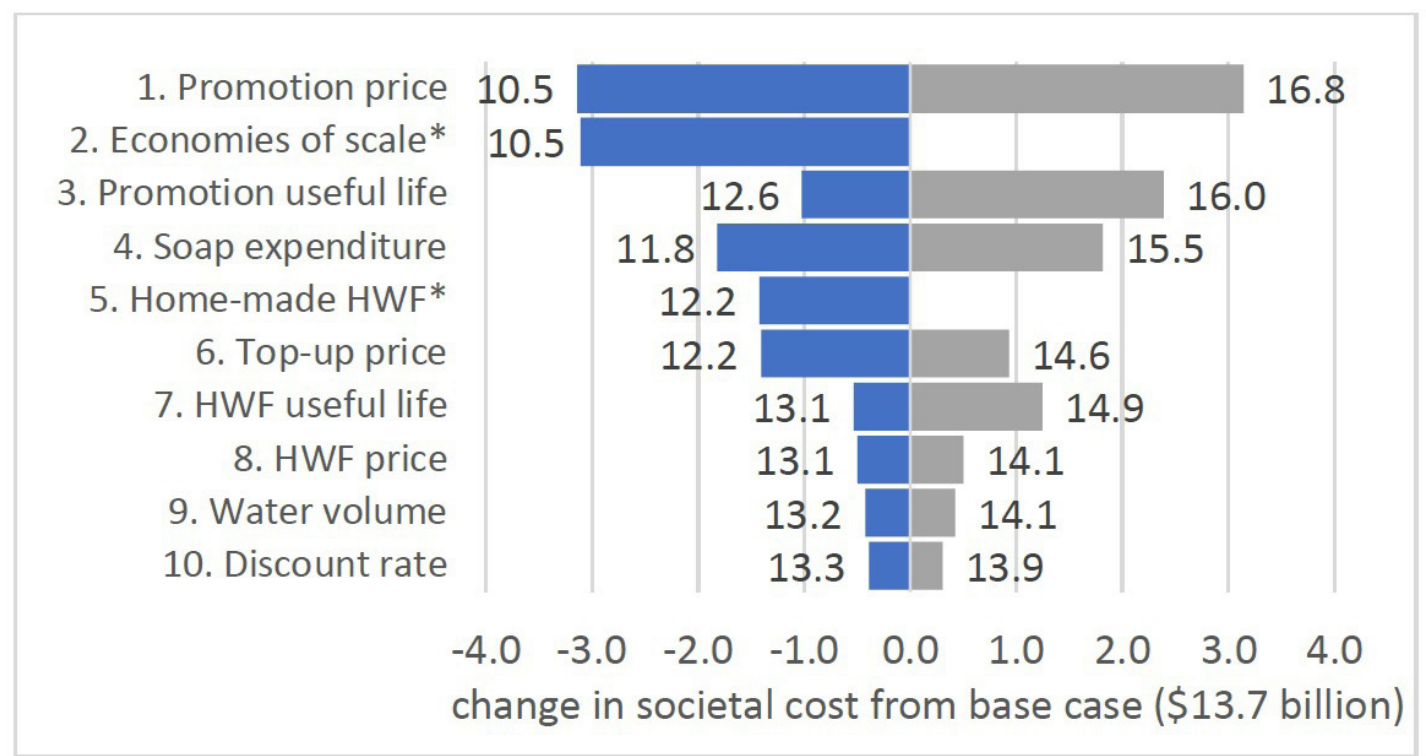

Figure 4 Tornado plot for total economic cost. Bars represent lower and upper estimates of total societal cost when parameters are at high and low plausible values. Parameters with the largest bars contribute most to uncertainty. *No upper estimate. HWF, handwashing facility. 
probabilistic sensitivity analysis is a robust way of characterising uncertainty and heterogeneity in synthesising data from diverse interventions and contexts. Our study's results can provide an empirical basis for country-level discussions of hand hygiene intervention prioritisation, costs and how to cover them, which are increasingly taking place in light of the COVID-19 pandemic. ${ }^{6}$ To this end, WHO and UNICEF have developed an easy-to-use costing tool based on our model, which provides countrylevel estimates, and makes assumptions and input data easily editable by the user to reflect local realities. ${ }^{41}$ It also allows estimation of costs for over 100 countries, not only LDCs.

Assuming that governments would fund promotion, top-up promotion and formative research, the average annual cost was US $\$ 0.47$ per head of LDC population. This represents $4.7 \%$ of median government health expenditure in LDCs (US $\$ 10.0$ per capita), ${ }^{42}$ and $12.3 \%$ of median WASH budgets of 23 LDCs (US\$3.8 per capita). ${ }^{43}$ The annual total represents $1 \%$ of the US $\$ 57$ billion in aid disbursed to LDCs by 'official donors' in 2019. ${ }^{44}$ Such expenditure may be justified on public health grounds since a substantial disease burden could be averted by hand hygiene, ${ }^{3}$ but better cost-effectiveness evidence would strengthen the case for health audiences. With only $16 \%$ of countries able to report the size of hygiene budgets or expenditures, ${ }^{13}$ wider application of the TrackFin methodology for estimating WASH expenditure would be beneficial. ${ }^{45}$ While the costs of HWFs, soap and water are borne by households in the majority of cases, they can also be subsidised directly or indirectly (eg, specific subsidies, cash transfers or humanitarian relief). The affordability of these costs for households requires investigation across a range of countries and settings. ${ }^{46}$ Soap presents a particular challenge, with survey data pointing to a strong socioeconomic gradient in soap availability within households. ${ }^{14}$ The median annual cost per household of US $\$ 17$ for soap is likely to be unaffordable for the poorest households, and further innovation in handwashing technologies that reduce amounts of soap and water needed is required. ${ }^{47}{ }^{48}$ Stimulation of soap markets in countries with particularly high prices would also be beneficial.

The headline hygiene-specific results of the previous global costing study ${ }^{10}$ are not directly comparable to our results, due to its reporting of hygiene-specific results for capital only and being for 140 countries. A comparison of unit prices is possible. Analysis of the earlier study's raw price data ${ }^{49}$ indicates a median total capital cost of US $\$ 5-$ US\$6 per person targeted (2015 prices), with software/ promotion accounting for two thirds of the total. ${ }^{50}$ The median total capital cost in our study is US $\$ 9.9$ per person targeted (2019 prices), with promotion accounting for $66 \%$ of the total. This suggests higher underlying prices, driven by: (1) our specification of a purpose-built HWF (when the earlier study's price data included many basic types); and (2) our inclusion of price data only for interventions including one-to-one promotion in the base case (when the earlier study pooled all types of intervention). The previous study's median recurrent cost was US\$2-US\$3 per person per year, which the underlying data suggest account for soap costs only. The equivalent for our study is US $\$ 6.3$ (of which soap accounts for $56 \%$, water $19 \%$ and top-up promotion $26 \%$ ). Since water was accounted for separately in the previous study, not as part of the hygiene total, our higher recurrent cost estimate appears to be accounted for by having included top-up promotion.

Water is an important recurrent economic cost of handwashing regardless of whether it is paid for. Capital investment in water infrastructure will also be required to enable handwashing in many settings. In LDCs, 351 million people use a 'less than basic' water supply, of which 141 million have a 'limited' service comprising an improved infrastructure at greater than $30 \mathrm{~min}$ round trip. ${ }^{4}$ The less water there is easily available in the household, the lower the likelihood of handwashing taking place, which makes effective promotion harder. On-premises water supply that is available when needed is most likely to support handwashing, but only $37 \%$ of the LDC population have a 'safely-managed' drinking water service that meets these criteria. ${ }^{4}$ Of the 81 million LDC households with 'limited' hygiene, we estimated that $53 \%$ had no water available at the HWF at the time of survey. The World Bank estimated that universal 'basic' water supply for those without it would cost US $\$ 7$ billion per year and 'safely managed' water supply US $\$ 38$ billion per year. ${ }^{10}$ Adding this to our results, to estimate the full cost of enabling hand hygiene, would substantially increase resource requirements.

We modelled scale-up as occurring in 10 equal cohorts over 10 years, but governments might decide to roll out interventions differently, for example, as a national campaign like the Swachh Bharat Mission for sanitation in India. ${ }^{51}$ This would lead to more front-loading of costs, but likely also to economies of scale and scope. Our deterministic sensitivity scenario assuming annual reductions in unit prices for promotion, HWFs and soap (to a total of $30 \%$ on year 1 prices by the seventh year of implementation) sees total and provider costs reduce substantially (figure 4 and online supplemental material D). In addition, of the 14 promotion price datapoints, only 6 were for interventions reaching more than 10000 households. Our input price data may therefore overestimate what it would cost to deliver interventions at large scale. Promotion price was the largest source of uncertainty (figure 4), and the range of our input price distribution for promotion was very wide (figure 1 ).

There may be efficiencies in combining handwashing messages with other messages, for example, within WASHspecific programmes or broader programmes such as those delivered by health extension workers (HEWs) ${ }^{52}$ Of the time Ethiopian HEWs spend providing health education and services, they spend $30 \%$ of it on hygiene and environmental sanitation. ${ }^{53}$ However, this includes messaging on water management, faeces disposal, hand 
hygiene, food hygiene, waste management, etc, so actual HEW time spent on hand hygiene promotion is likely to be small. When messaging is diluted in this way there may be lower effectiveness than standalone campaigns, but this requires investigation. In addition, in some countries and settings community health workers are overstretched and lack the skills, training and support needed to deliver behaviour change programmes effectively. ${ }^{54-56}$

Following norms in resource requirement estimation, ${ }^{12}$ we estimate the cost of reaching all target households. Therefore, uptake and adherence are not accounted for, as would be necessary in a cost-benefit or costeffectiveness analysis. As with many public health interventions, uptake of handwashing behaviours as a result of an intervention is typically below $50 \%$, as is subsequent adherence. ${ }^{20}{ }^{57}$ Even having a HWF with soap and water is no guarantee that hand hygiene is practised correctly at critical times, and promotion may benefit households already with a basic hygiene service. ${ }^{5}$ In the main analysis, we costed interventions only for households with 'no hygiene facility'. This was with a view to estimating the order of magnitude of costs, rather than suggesting that such households are specifically targeted by programmes. Since promotion costs almost quadruple if the whole population receives the intervention, there may be a balance to be sought. Some modes of delivery might be provided to whole populations, with others only being used in specific high-risk areas. For example, georeferenced data from national health surveys could be used to identify sub-national areas with low HWF ownership or high cholera incidence. ${ }^{58}$

Taking into account the above considerations, the cost of achieving universal hand hygiene behaviour, as opposed to universal access to basic hygiene services, is likely to be larger than our headline estimate. Poor-quality cost evidence is a barrier to scaling up public health interventions and ${ }^{89}$ with some notable exceptions, ${ }^{21}{ }^{32}$ the costs of specific handwashing promotion interventions are rarely systematically collected. The costs of hand hygiene in healthcare facilities also require further invesigation. ${ }^{59}$ Impact evaluations need to collect intervention cost data if they are to effectively inform priority-setting, ${ }^{60}{ }^{61}$ but we received usable cost data from fewer than half of the investigators contacted for this study. Almost all studies included in our model reported or necessitated topdown retrospective analyses, an approach with substantial limitations, ${ }^{62}$ and none were peer-reviewed studies employing bottom-up prospective costing. ${ }^{63}$ In addition, more studies and meta-analyses are required on the relative effectiveness of different approaches to hand hygiene promotion, which would also enable more interventionspecific costing. ${ }^{20}$ For example, handwashing campaigns increasingly include social media components, ${ }^{6465}$ but minimal data are available on the costs and effectiveness of such strategies.

Limitations of our study are as follows. First, though we account for a higher economic cost of water for people without an on-premises piped supply, challenges of limited water availability for handwashing are not incorporated in any other way. Second, while we distinguish between hygiene service level differences between urban and rural areas, we apply a single national price per cost category, in the absence of reliable data on how prices of promotion, HWFs, soap etc vary by urban/rural setting. Prices of many inputs are likely to be higher in rural areas further from markets, and income poverty is predominantly rural. ${ }^{66}$ Third, while we clearly specified the assumed intervention and extracted intervention characteristics from studies, the data underlying the sample mean for promotion price derive from many types of interventions at different scales. Fourth, follow-up formative research to revise promotion interventions based on implementation experience was not included. This was because many factors would determine the nature, timing and scale of such activities, and the cost would be small relative to other categories.

\section{CONCLUSION}

We estimated the economic costs of universal access to basic hand hygiene services in household settings in 46 LDCs, finding that US $\$ 12.2-U S \$ 15.3$ billion is needed over 10 years. The promotion costs within this are US $\$ 5.7$ billion, representing $4.7 \%$ of median government health expenditure in LDCs, and $1 \%$ of their aid receipts. The remainder comprises HWFs (US $\$ 1.7$ billion), soap (US $\$ 5.0$ billion) and water (US $\$ 1.3$ billion). These costs could be covered by mobilising resources from across government and partners, and could be reduced by harnessing economies of scale and integrating hand hygiene with other behavioural change campaigns where appropriate. Innovation is required to make soap more affordable and available for the poorest households. Better evidence on the relative costs, effectiveness and costeffectiveness of different promotion interventions for uptake and adherence of hand hygiene behaviours would enable more efficient investments.

Twitter lan Ross @ianrossuk

Acknowledgements We acknowledge the time and effort of impact evaluation investigators in answering our questions and providing data. We also acknowledge the advice and support of the steering committee, and seminar participants at LSHTM's economic evaluation group and environmental health group.

Contributors IR developed the methods with support from JEM, MM and RD. RJ, $\mathrm{TS}, \mathrm{GH}$ and RD provided data and analysis supporting methodological decisions and interpretation. IR searched for and extracted cost data. IR and JEM extracted intervention characteristics data. IR developed the model, analysed data, and is guarantor of the study. All authors inputted into the manuscript.

Funding This work was funded by WHO (with support from the Governments of Japan, UK and The Netherlands) and UNICEF.

Competing interests A portion of RD's salary is supported through an unrestricted donation to LSHTM by Reckitt, exclusive of the work on this manuscript.

Patient consent for publication Not applicable.

Ethics approval Ethical approval was not required because the study analysed secondary data from published studies.

Provenance and peer review Not commissioned; externally peer reviewed. 
Data availability statement Data are available in a public, open access repository. All data relevant to the study are included in the article or uploaded as online supplemental information. Quantity data are derived from public domain resources (https://washdata.org/). Price data are derived from published studies referenced in the manuscript. Unit prices are tabulated in online supplemental material.

Supplemental material This content has been supplied by the author(s). It has not been vetted by BMJ Publishing Group Limited (BMJ) and may not have been peer-reviewed. Any opinions or recommendations discussed are solely those of the author(s) and are not endorsed by BMJ. BMJ disclaims all liability and responsibility arising from any reliance placed on the content. Where the content includes any translated material, BMJ does not warrant the accuracy and reliability of the translations (including but not limited to local regulations, clinical guidelines, terminology, drug names and drug dosages), and is not responsible for any error and/or omissions arising from translation and adaptation or otherwise.

Open access This is an open access article distributed in accordance with the Creative Commons Attribution 4.0 Unported (CC BY 4.0) license, which permits others to copy, redistribute, remix, transform and build upon this work for any purpose, provided the original work is properly cited, a link to the licence is given, and indication of whether changes were made. See: https://creativecommons.org/ licenses/by/4.0/.

ORCID iD

lan Ross http://orcid.org/0000-0002-2218-5400

\section{REFERENCES}

1 Wolf J, Hunter PR, Freeman MC, et al. Impact of drinking water, sanitation and handwashing with soap on childhood diarrhoeal disease: updated meta-analysis and meta-regression. Trop Med Int Health 2018;23:508-25.

2 McGuinness SL, Barker SF, O'Toole J, et al. Effect of hygiene interventions on acute respiratory infections in childcare, school and domestic settings in low- and middle-income countries: a systematic review. Trop Med Int Health 2018;23:816-33.

3 Prüss-Ustün A, Wolf J, Bartram J, et al. Burden of disease from inadequate water, sanitation and hygiene for selected adverse health outcomes: an updated analysis with a focus on low- and middleincome countries. Int J Hyg Environ Health 2019;222:765-77.

4 WHO \& UNICEF. Progress on household drinking water, sanitation and hygiene 2000-2020 five years into the SDGs. Geneva, 2021. https://washdata.org/sites/default/files/2021-06/jmp-2021-washhouseholds-LAUNCH-VERSION.pdf

5 Wolf J, Johnston R, Freeman MC, et al. Handwashing with soap after potential faecal contact: global, regional and country estimates. Int J Epidemiol 2019;48:1204-18.

6 WHO \& UNICEF. Hand hygiene for all, 2020.

7 Cairncross S, Feachem RG. Environmental health engineering in the tropics : an introductory text. 1st edn. Wiley, 1983.

8 Milat AJ, Bauman A, Redman S. Narrative review of models and success factors for scaling up public health interventions. Implement Sci 2015:1-11.

9 Subramanian S, Naimoli J, Matsubayashi T, et al. Do we have the right models for scaling up health services to achieve the millennium development goals? BMC Health Serv Res 2011;11:336.

10 Hutton G, Varughese M. The costs of meeting the 2030 sustainable development goal targets on drinking water, sanitation, and hygiene. World Bank; 2016. https://openknowledge.worldbank.org/handle/ 10986/23681

11 Hutton G. Editorial: can we meet the costs of achieving safely managed drinking-water, sanitation and hygiene services under the new sustainable development goals? J Water Sanit Hyg Dev 2016;6:191-4.

12 Vassall A, Sweeney S, Kahn JG. Reference case for estimating the costs of global health services and interventions 2017.

13 WHO. Hygiene: UN-Water GLAAS findings on national policies, plans, targets and finance, 2020. Available: https://www.unwater.org/ publications/hygiene-un-water-glaas-findings-on-national-policiesplans-targets-and-finance/

14 Kumar S, Loughnan L, Luyendijk R, et al. Handwashing in 51 countries: analysis of proxy measures of handwashing behavior in multiple indicator cluster surveys and demographic and health surveys, 2010-2013. Am J Trop Med Hyg 2017;97:447-59.

15 UN. List of least developed countries (as of 11 February 2021), 2021 Available: https://www.un.org/development/desa/dpad/wp-content/ uploads/sites/45/publication/ldc_list.pdf
16 WHO \& UNICEF. Dataset for WASH in households - July 2021 version, 2021. Available: https://washdata.org/data

17 DHS. DHS program STATcompiler, 2021.

18 UN-DESA. World population prospects 2019, 2019. Available: https://population.un.org/wpp/ [Accessed 8 Jun 2021].

19 Ejemot-Nwadiaro RI, Ehiri JE, Arikpo D, et al. Hand-washing promotion for preventing diarrhoea. Cochrane Database Syst Rev 2021;12:CD004265.

20 De Buck E, Van Remoortel H, Hannes K, et al. Approaches to promote handwashing and sanitation behaviour change in lowand middle-income countries: a mixed method systematic review. Campbell Syst Rev 2017;13:1-447.

21 Borghi J, Guinness L, Ouedraogo J, et al. Is hygiene promotion cost-effective? A case study in Burkina Faso. Trop Med Int Health 2002;7:960-9.

22 Bikash Srot Kendra. Piloting hygiene promotion through routine immunisation in Nepal, 2017.

23 Pinfold JV, Horan NJ. Measuring the effect of a hygiene behaviour intervention by indicators of behaviour and diarrhoeal disease. Trans $R$ Soc Trop Med Hyg 1996;90:366-71.

24 Freeman MC, Delea MG, Snyder JS. The impact of a demand-side sanitation and hygiene promotion intervention on sustained behavior change and health in Amhara, Ethiopia : a cluster-randomized trial. medRxiv 2021.

25 Rajaraman D, Varadharajan KS, Greenland K, et al. Implementing effective hygiene promotion: lessons from the process evaluation of an intervention to promote handwashing with soap in rural India. BMC Public Health 2014:14:1179.

26 Saadé C, Bateman M, Bendahmane DB. The story of a successful public-private partnership in central America. handwashing for diarrheal disease prevention. Arlington, Virginia: Basic Support for Child Survival Project (BASICS II), 2001

27 Greenland K, Chipungu J, Curtis V, et al. Multiple behaviour change intervention for diarrhoea control in Lusaka, Zambia: a cluster randomised trial. Lancet Glob Health 2016;4:e966-77.

28 Evans B, Bates L, Halder A. Analysing the value for money of SHEWA-B in Bangladesh, 2015.

29 Waterkeyn J, Matimati R, Muringaniza A. Comparative assessment of hygiene behaviour change and cost-effectiveness of community health clubs in Rwanda and Zimbabwe. Healthc Access - Reg Overview 2019.

30 Biran A, White S, Awe B, et al. A cluster-randomised trial to evaluate an intervention to promote handwashing in rural Nigeria. Int $\mathrm{J}$ Environ Health Res 2020:1-16.

31 George CM, Monira S, Sack DA, et al. Randomized controlled trial of hospital-based hygiene and water treatment intervention $(\mathrm{CHoBI})$ to reduce cholera. Emerg Infect Dis 2016;22:233-41.

32 Briceño B, Chase C. Cost and cost-efficiency of rural sanitation and handwashing promotion: activity-based costing and experimental evidence from Indonesia, India, Tanzania and Peru. World Bank; 2014.

33 IBNET. IBNET tariffs database. Int. Benchmarking Netw. Water Sanit. Util, 2021. Available: https://tariffs.ib-net.org/ [Accessed 15 Feb 2021].

34 Curtis V, Kanki B, Cousens S, et al. Dirt and diarrhoea: formative research in hygiene promotion programmes. Health Policy Plan 1997;12:122-31.

35 Pickering AJ, Null C, Winch PJ, et al. The WASH benefits and SHINE trials: interpretation of WASH intervention effects on linear growth and diarrhoea. Lancet Glob Health 2019;7:e1139-46.

36 Hutton $\mathrm{G}$, Chase $\mathrm{C}$. The knowledge base for achieving the sustainable development goal targets on water supply, sanitation and hygiene. Int J Environ Res Public Health 2016;13 doi:10.3390/ ijerph13060536

37 Wilkinson T, Sculpher MJ, Claxton K, et al. The International decision support initiative reference case for economic evaluation: an aid to thought. Value Health 2016;19:921-8.

38 Turner HC, Lauer JA, Tran BX, et al. Adjusting for inflation and currency changes within health economic studies. Value Health 2019;22:1026-32.

39 World Bank. PPP conversion factor, GDP (LCU per international \$, 2021. https://data.worldbank.org/indicator/PA.NUS.PPP

40 Briggs A, Sculpher M, Claxton K. Decision modelling for health economic evaluation. Oxford, UK: OUP, 2006.

41 WHO \& UNICEF. Costing tool for estimating the cost of interventions to improve hand hygiene in domestic settings. WHO/HEP/ECH/ WSH/2021.3, 2021. Available: https://www.who.int/publications/m/ item/WHO-HEP-ECH-WSH-2021.3

42 WHO. Domestic general government health expenditure (GGHE-D) per Capita in US\$. Glob. Heal. Expend. Database, 2021. Available: https://apps.who.int/nha/database [Accessed 8 Jun 2021]. 
43 WHO. National systems to support drinking-water, sanitation and hygiene: global status report 2019, 2019. https://www.unwater.org/ publications/un-water-glaas-2019-national-systems-to-supportdrinking-water-sanitation-and-hygiene-global-status-report-2019/

44 OECD. Creditor reporting system, 2021. Available: https://stats. oecd.org/Index.aspx?DataSetCode=crs1\# [Accessed 24 Jul 2021].

45 WHO. Reflecting on TrackFin 2012-2020: key results, lessons learned and the way forward. Geneva, 2021. https://apps.who.int/ iris/handle/10665/341793

46 UNICEF \& WHO. The measurement and monitoring of water supply, sanitation and hygiene (WASH) affordability: a missing element of monitoring of sustainable development goal (SDG) targets 6.1 and 6.2. New York, 2021. Available: https://apps.who.int/iris/rest/ bitstreams/1344432/retrieve

47 Whinnery J, Penakalapati G, Steinacher R, et al. Handwashing with a water-efficient tap and low-cost foaming soap: the Povu Poa 'Cool Foam' system in Kenya. Glob Health Sci Pract 2016;4:336-41. doi:10.9745/GHSP-D-16-00022

48 Ashraf S, Nizame FA, Islam M, et al. Nonrandomized trial of feasibility and acceptability of strategies for promotion of Soapy water as a handwashing agent in rural Bangladesh. Am J Trop Med Hyg 2017;96:421-9.

49 World Bank. Raw dataset - the costs of meeting the 2030 SDG targets on drinking water, sanitation, and hygiene, 2016. Available: https://datacatalog.worldbank.org/dataset/costs-meeting-2030-sdgtargets-drinking-water-sanitation-and-hygiene [Accessed 16 Dec 2020].

50 Ross I. Summary report on the economics of hygiene programmes, 2020. hygienehub.info

51 Curtis V. Explaining the outcomes of the 'Clean India' campaign: institutional behaviour and sanitation transformation in India. BMJ Glob Health 2019;4:e001892 doi:10.1136/bmjgh-2019-001892

52 Perry HB, Zulliger R, Rogers MM. Community health workers in lowmiddle-, and high-income countries: an overview of their history, recent evolution, and current effectiveness. Annu Rev Public Health 2014;35:399-421.

53 Tilahun $\mathrm{H}$, Fekadu B, Abdisa $\mathrm{H}$, et al. Ethiopia's health extension workers use of work time on duty: time and motion study. Health Policy Plan 2017;32:320-8.

54 Aseyo RE, Mumma J, Scott K, et al. Realities and experiences of community health volunteers as agents for behaviour change: evidence from an informal urban settlement in Kisumu, Kenya. Hum Resour Health 2018;16:1-12. doi:10.1186/s12960-018-03184
55 Suri A, Gan K, Carpenter S. Voices from the field: perspectives from community health workers on health care delivery in rural KwaZulu-Natal, South Africa. J Infect Dis 2007;196:S505-11. doi:10.1086/521122

56 Cunningham K, Suresh S, Kjeldsberg C, et al. From didactic to personalized health and nutrition counselling: a mixed-methods review of the GALIDRAA approach in Nepal. Matern Child Nutr 2019;15:1-8.

57 Whittington D, Jeuland M, Barker K, et al. Setting priorities, targeting subsidies among water, sanitation, and preventive health interventions in developing countries. World Dev 2012;40:1546-68. doi:10.1016/j.worlddev.2012.03.004

58 Lessler J, Moore SM, Luquero FJ. Articles Mapping the burden of cholera in sub-Saharan Africa and implications for control : an analysis of data across geographical scales. Lancet 2018;6736:1-8.

59 Chaitkin M, McCormick S, Alvarez-Sala Torreano J, et al. Estimating the cost of achieving universal basic wash standards in health care facilities in the 46 least developed countries. SSRN Journal 2021. doi:10.2139/ssrn.3858515

60 J-PAL. J-PAL costing guidelines, 2016. Available: https://www. povertyactionlab.org/sites/default/files/resources/J-PAL Costing Guidelines 2016.05.pdf

61 Whittington D, Radin M, Jeuland M. Evidence-based policy analysis? The strange case of the randomized controlled trials of communityled total sanitation. Oxford Rev Econ Policy 2020;36:191-221.

62 Chapko MK, Liu C-F, Perkins M, et al. Equivalence of two healthcare costing methods: bottom-up and top-down. Health Econ 2009;18:1188-201.

63 Crocker J, Saywell D, Shields KF, et al. The true costs of participatory sanitation: evidence from community-led total sanitation studies in Ghana and Ethiopia. Sci Total Environ 2017;601-602:1075-83.

64 Moktan PS, Bajracharya B. Using social media in the local language to engage with populations in a tailored way COVID-19. Hyg Hub 2020 https://resources.hygienehub.info/en/articles/4247875-usingsocial-media-in-the-local-language-to-engage-with-populations-ina-tailored-way

65 Fung IC-H, Cai J, Hao Y, et al. Global handwashing day 2012: a qualitative content analysis of Chinese social media reaction to a health promotion event. Western Pac Surveill Response $J$ 2015;6:34-42.

66 World Bank. Poverty and shared prosperity 2020: reversals of fortune. Washington, DC, 2020. https://www.worldbank.org/en/ publication/poverty-and-shared-prosperity 